\title{
Delayed Neurological Sequelae Following Carbon Monoxide Poisoning and Skin Graft: A Case Report
}

\author{
Reza Bidaki ${ }^{1,2}$, Azadeh Rahavii, ${ }^{1 *}$, Farzaneh Dehghani ${ }^{1}$, Mohamad Ebrahim Ghanei ${ }^{3}$, Najmeh Zaer- \\ Alhosseini ${ }^{4}$, Maryam Khorasani ${ }^{5}$
}

\author{
${ }^{1}$ Department of Psychiatry, Research Center of Addiction and Behavioral Sciences, Shahid Sadoughi University of \\ Medical Sciences, Yazd, Iran \\ ${ }^{2}$ Diabetes Research Center, Shahid Sadoughi University of Medical Sciences, Yazd, Iran \\ ${ }^{3}$ Department of Radiology, School of Medicine, Shahid Sadoughi University of Medical Sciences, Yazd, Iran \\ ${ }^{4}$ Science and Research Branch, Islamic Azad University, Yazd, Iran \\ ${ }^{5}$ Department of Psychology, University of Shihid Chamran, Ahvaz, Iran
}

*Corresponding Author: Azadeh Rahavi, M.D., Psychiatrist, Research Center of Addiction and Behavioral Sciences, Shahid Sadoughi University of Medical Sciences, Yazd, Iran. Tel: +98-9131578581, Email: azadehrahavi@gmail.com

Received May 30, 2020; Accepted November 2, 2020; Online Published November 18, 2020

\begin{abstract}
Introduction: Carbon monoxide (CO) poisoning is a prevalent lethal condition. The clinical feature of this type of poisoning varies from headache and nausea to more severe conditions. After recovery from the acute intoxication, neurological or behavioral problems may emerge. In 3\%-40\% of cases, delayed neuropsychiatric syndrome (DNS) in post CO poisoning, generally develops within few weeks after a preliminary remission from acute poisoning.

Case Presentation: We report a patient with relatively suitable premorbid. He was admitted to the hospital with fire burning, co poisoning and discharged home with good general conditions and mental status by receiving normal baric oxygen $100 \%$. later in post-operative management of skin graft he developed a fulminant neurological deficit by impaired memory and concentration, loosening of association, disorientation to place, time and person, agitation, aggression, mood labiality, urinary incontinency and encopresis, slow psychomotor retardation, false and approximate answers to questions, auditory and visual hallucination, staring and inappropriate laughing. As the patient was not responsive to neurological treatment, he was referred to psychiatric service. DNS in this patient resolved gradually during a short period of psychopharmacotherapy and supportive psychotherapy Conclusion: CO poisoning may lead to neuropsychiatric sequel and neuroimaging changes which could be reversible. Keywords: Carbon Monoxide Poisoning, Neurological Syndrome, Cognitive Impairment
\end{abstract}

\section{Introduction}

Carbon monoxide (CO) poisoning is a prevalent threatening status. ${ }^{1,2}$ Carbon derived fuel is the most common source for Iranian to supply home heating during winter. ${ }^{3}$

The clinical feature of $\mathrm{CO}$ poisoning varies from headache and nausea to more severe conditions consist of lethargy, loss of consciousness, seizure and death. ${ }^{4,5}$ After recovery from acute intoxication, neurological behavioral manifestation may emerge in form of Delayed neuropsychiatric syndrome (DNS). DNS generally develops within a few weeks after a preliminary remission of acute poisoning in 3\%-40\% of cases. ${ }^{6,7}$ Contemporary treatment for patients with acute CO poisoning is 100 percent normobaric oxygen or hyperbaric-oxygen therapy (HBO2). ${ }^{4}$ However, there is no well-established treatment for CO-associated DNS. ${ }^{8}$ This signifies a need to find efficient treatment for this devastating condition

In this case report, we represent a patient with $\mathrm{CO}$ poisoning who developed DNS following skin graft surgery. Although the neuroimaging reports indicated irreversible changes and the patient prognosis was considered poor, to our surprise the patient's cognitive sequelae improved gradually within minimal intervention during few weeks of psychiatric treatment.

\section{Case Presentation}

Hereby, we report a patient with relatively suitable premorbid. He was admitted to the hospital in December 2016 with fire burning and co poisoning. In parallel with atrial blood gas $(\mathrm{ABG})$ data (Table 1), the diagnosis of $\mathrm{CO}$ poisoning was highly probable and accordingly normal baric oxygen $100 \%$ administered straight away. He gradually could obtain his normal status as burns management and serum therapy were also running during 2 days of hospitalization. Eventually, he discharged from hospital conscious and alert with suitable general medical condition.

Copyright $\odot 2020$ The Author(s). This is an open-access article distributed under the terms of the Creative Commons Attribution License (http:// creativecommons.org/licenses/by/4.0), which permits unrestricted use, distribution, and reproduction in any medium, provided the original work is properly cited. 


\begin{tabular}{ll} 
Table 1. Atrial Blood Gas (ABG) data \\
\hline Base Excess & 0.3 \\
\hline $\mathbf{p H}$ & 7.47 \\
\hline Hco3 & $23.2 \mathrm{meq} / \mathrm{L}$ \\
\hline PCO & $32 \mathrm{~mm} \mathrm{Hg}$ \\
\hline PCO2 & $32 \mathrm{~mm} \mathrm{Hg}$ \\
\hline PO2 & $70 \mathrm{~mm} \mathrm{Hg}$ \\
\hline
\end{tabular}

Four weeks after being discharged, the patient returned back to hospital for unhealed burns with no other evident complaint. As a result, he hospitalized for skin graft surgery. Twenty-four hours after general anesthesia, he developed cognition decline gradually by impairment in memory and concentration, loosening of association, disorientation to place, time and person, agitation, aggression, mood labiality, urinary incontinence, encopresis, psychomotor retardation, inappropriate laughing, staring, auditory and visual hallucination and wrong and approximate answers to questions, prosopagnosia agraphestesia, topographagnosia. There was rigidity, tremor and positive primitive reflexes (i.e. grasp reflex) in neurological examination.

This situation, describing a fulminant neurological process, brought psychiatry and neurology consultation services to work up the patient with more psycho-cognitive and paraclinic investigations.

Psycho-cognitive assessments such as Wechsler Memory Scale (WMS), Mini-Mental State Examination (MMSE), Rey-Osterrieth complex figure test (ROCF) and BenderGestalt test were performed. WMS, as a neuropsychological test to measure different memory functions, illustrated the memory quotient of 62 in our patient as an abnormal score. The other test applied to check memory was MMSE. This test is used extensively to measure cognitive impairment. With its 30-point any score greater than or equal to (24 out of 30 points) is considered as a normal cognition. In our patients, the score of 7 as sever cognitive impairment was yielded. To check visual memory, he could not accomplish; neither copy nor recalling task in ROCF and BenderGestalt test.

Brain imaging findings including diffusion-weighted magnetic resonance imaging (MRI) showed high signal intensity in cortex and white matter of both fronto-parietal and rolandic cortices (Figure 1).

In ADC maps MRI (Figure 2) low signal intensity in corresponding Diffusion-weighted abnormalities was seen that indicates acute ischemia. Axial T2W brain MRI demonstrated abnormal high signal intensity in subcortical and white matter of parasagittal watershed region. In computed tomography (CT) scan diffuse edema with effacement of the cerebrospinal fluid-containing spaces and decreased cortical gray matter attenuation with loss of gray-white differentiation and decreased bilateral basal ganglia attenuation was seen. In total, evidences in brain CT and MRI depicted acute cerebral ischemia that is in parallel with ABG findings and its occurrence following

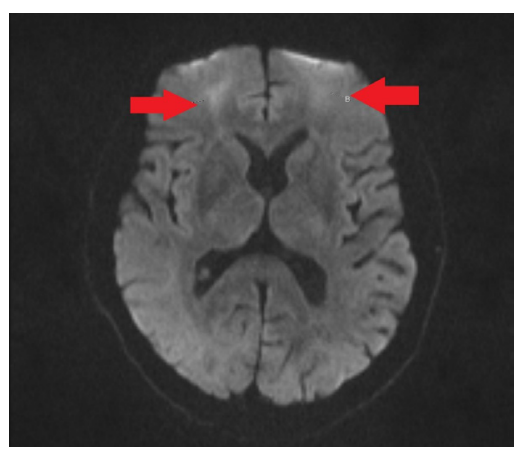

Figure 1. Diffusion-Weighted Magnetic Resonance Imaging (MRI): High Signal Intensity in Cortex and White Matter of Both Fronto-Parietal and Rolandic Cortices.

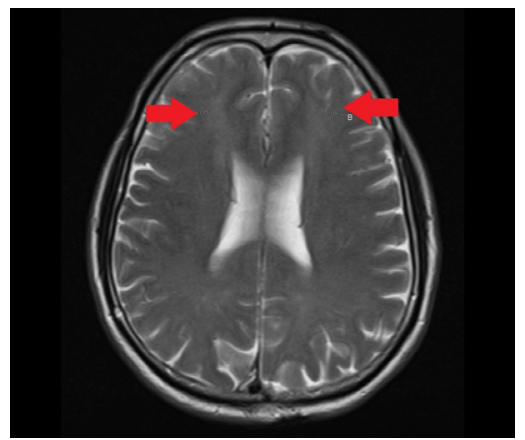

Figure 2. ADC Maps MRI: Low Signal Intensity in Corresponding DiffusionWeighted Abnormalities.

\section{CO poisoning.}

To comprehend the mechanism of symptoms and also localization of cognitive disorders ex. Dementia, prosopagnosia, psychomotor retardation, apathy and poverty of thought we requested for quantitative electroencephalography (QEEG). Presumptive diagnosis reported that findings may relate to depression, poor concentration and anxiety problem. Frequency band abnormalities (alpha and theta band) have found promise as have combinatorial measures such as cordance. Perhaps there is a previous damage to diffuse areas (Box 1).

Through diagnosis of DNS induced by CO poisoning, the patient received normobaric oxygen therapy; because of psychotic symptoms we ordered Haloperidol $0.5 \mathrm{mg}$ II $\mathrm{BID}$, and in order to improve memory function we prescribed memantine and piracetam. For reducing anxiousness we prescribed Tab. propranolol $10 \mathrm{mg}$ BID and Tab. trazodone $50 \mathrm{mg}$ at night.

After psychiatric treatment, the patient did not show

\section{Box 1. QEEG Findings}

- Enhanced delta and theta in diffused regions are artifact

- Enhanced alpha in anterior area

- Increase of high beta in central

- Depletion of relative beta

- LORETA 3 dimensional source analysis showed excessive current sources in temporo frontal with maximum at Delta and Theta. High Beta $(25 \mathrm{~Hz})$ in posterior 
significant improvement in level of cognition. In the follow up, nearly after one month of being discharged, cognitive signs and symptoms completely resolved in a way he could obtain his previous personal and occupational function. The Only complaint was about estimating the distance specially during driving. He had euthymic mood yet there was a hesitancy and uncertainty for him in decision making and initiation of works. At the moment, he is back to his job as a technician in a health care center in the section for diagnosis of leishmaniasis. He has normal personal function; he can drive, with no anxiousness and fear. Neurological examinations and memory functioning are normal.

\section{Discussion}

Accidental poisoning through inhalation of toxic gases is not rare $^{9}$ and sometime they may lead to irreversible side effects.

In this study, we represented a patient who did not demonstrate serious complications immediately after CO poisoning but later when the skin graft surgery was done he developed severe cognitive and perceptional impairments with dysphoric mood, prosopagnosia, agraphestesia, geographesthesia. According to such clinical manifestation, the prognosis of our patient was not predictable and the course of the disease was not obvious. Imaging findings were also indicative of severe and serious complications in a way neurologists considered an irreversible complication for the patient.

However, the course of the disease initially started with waxed and waned nature and later during a month after discharge he recovered dramatically and obtained normal personal, social and occupational function while receiving haloperidol, memantine and piracetam. The only residual symptom that did not recovered was the patient's disability in estimating distances which sometimes would bring difficulty for him to drive or park his car.

In order to prevent delayed cognitive sequelae, alternatives like anti-oxidants, steroids, erythropoietin, and other potential therapies have been suggested. ${ }^{8}$ These contraptions have been supported by several clinical experiences and scientific investigations. A recent singlecase study showed promise of combined application of methylprednisolone pulse and memantine hydrochloride in the improvement dNS. ${ }^{10}$ Knowing side effect of corticosteroids on exacerbation of psychotic symptoms and mood imbalance, in our case we did not order prednisolone.

Despite several studies emphasizing on the treatment of DNS, Weaver et al in a clinical trial study declared that $\mathrm{HBO} 2$ significantly reduces the risk for cognitive sequela as a mean to prevent DNS. ${ }^{7}$ Accordingly, in a study evaluating neuropsychological in children and adolescents after $\mathrm{CO}$ poisoning suggested that the duration of $\mathrm{CO}$ exposure, history of loss of consciousness, number of sessions receiving hyperbaric oxygen therapy influence the cognitive symptoms levels. On the other hand carboxyhemoglobin level found to be not correlated with later neuropsychiatric test scores. However, hyper baric oxygen therapy has been shown to be the most effective therapy in curing cognitive complications induced by $\mathrm{CO}$ poisoning. ${ }^{11,12}$ In our study, since hyperbaric oxygen was not available, we applied normobaric oxygen therapy for hours which accounts for one of limitation in our study.

It is also controversial whether complications in our patient generated subsequent to $\mathrm{CO}$ poisoning in the form of delayed sequela or as the side effect of anesthesia process for skin graft surgery or synergistic effect of both condition. To address this question clinical trials are needed to confirm the findings in our study.

\section{Conclusion}

CO poisoning may lead to delayed neuropsychiatric complications with irreversible appearance in neuroimaging. However, remission and resolve of symptoms could be possible by supportive interventions.

\section{Authors' Contributions}

In this manuscript $\mathrm{RB}, \mathrm{AR}$ and $\mathrm{FD}$ have co-written the manuscript; MEG, NZ, MK have collected data by performing psychiatry tests. RB has designed the study. $\mathrm{AR}$ and $\mathrm{RB}$ have interviewed with the patient and FD has submitted the paper.

\section{Conflict of Interest Disclosures}

The authors declare that they have no conflicts of interest.

\section{Ethical Approval}

We ensured patient health data privacy and obtained written informed consent from the patient and his family. All of the ethical issues were considered during interview and gathering data.

\section{Acknowledgments}

We appreciate the patient and his family's cooperation with our study.

\section{References}

1. Prockop LD, Chichkova RI. Carbon monoxide intoxication: an updated review. J Neurol Sci. 2007;262(1-2):122-130. doi:10.1016/j.jns.2007.06.037.

2. Bleecker ML. Carbon monoxide intoxication. Handb Clin Neurol. 2015;131:191-203. doi:10.1016/b978-0-444-626271.00024-x.

3. Hosseininejad SM, Aminiahidashti H, Goli Khatir I, Ghasempouri SK, Jabbari A, Khandashpour M. Carbon monoxide poisoning in Iran during 1999-2016: a systematic review and meta-analysis. J Forensic Leg Med. 2018;53:87-96. doi:10.1016/j.jflm.2017.11.008.

4. Rose JJ, Wang L, Xu Q, et al. Carbon monoxide poisoning: pathogenesis, management, and future directions of therapy. Am J Respir Crit Care Med. 2017;195(5):596-606. doi:10.1164/ rccm.201606-1275Cl.

5. Balzan MV, Agius G, Galea Debono A. Carbon monoxide poisoning: easy to treat but difficult to recognise. Postgrad Med J. 1996;72(850):470-473. doi:10.1136/pgmj.72.850.470. 
6. Pepe G, Castelli M, Nazerian P, et al. Delayed neuropsychological sequelae after carbon monoxide poisoning: predictive risk factors in the Emergency Department. A retrospective study. Scand J Trauma Resusc Emerg Med. 2011;19:16. doi:10.1186/1757-7241-19-16.

7. Weaver LK, Hopkins RO, Chan KJ, et al. Hyperbaric oxygen for acute carbon monoxide poisoning. $N$ Engl J Med. 2002;347(14):1057-1067. doi:10.1056/NEJMoa013121.

8. Oh S, Choi SC. Acute carbon monoxide poisoning and delayed neurological sequelae: a potential neuroprotection bundle therapy. Neural Regen Res. 2015;10(1):36-38. doi:10.4103/1673-5374.150644.

9. Matthews G. Toxic gases. Postgrad Med J. 1989;65(762):224232. doi:10.1136/pgmj.65.762.224.

10. Iwamoto K, Ikeda K, Mizumura S, Tachiki K, Yanagihashi
M, Iwasaki Y. Combined treatment of methylprednisolone pulse and memantine hydrochloride prompts recovery from neurological dysfunction and cerebral hypoperfusion in carbon monoxide poisoning: a case report. J Stroke Cerebrovasc Dis. 2014;23(3):592-595. doi:10.1016/j. jstrokecerebrovasdis.2013.05.014.

11. Vázquez-Lima MJ, Álvarez-Rodríguez C, Cruz-Landeira A, López-Rivadulla M. [Delayed neurological syndrome following carbon monoxide poisoning]. Rev Neurol. 2015;61(4):153158.

12. Neubauer RA, Neubauer V, Nu AKC, Maxfield WS. Treatment of late neurologic sequelae of carbon monoxide poisoning with hyperbaric oxygenation: a case series. J Am Physicians Surg. 2006;11(2):56-59. 